\title{
Unusual Evolution and Computerized Tomographic Appearance of a Gliosarcoma
}

\author{
Mark C. Preul, Jose A. Espinosa, Donatella Tampieri and Stirling Carpenter
}

\begin{abstract}
A patient with a remote infarct, seizures, mild hemiparesis, and dysphasia became obtunded over four months and died. Computerized tomography (CT) over 5 years showed a consistent, large, wedge-shaped left hemisphere hypodensity with a central calcification, but without signs of mass effect. This was interpreted as an infarct of the left middle cerebral artery territory. Post-mortem examination of the brain revealed the entire area appearing as infarct on CT was a gliosarcoma. We suspect that the unusual CT appearance of the lesion was likely caused by multiple pathologies: a low grade glioma transforming into a gliosarcoma that was able to spread throughout the area of infarct encephalomalacia without revealing a typical CT appearance of mass effect. The patient's brief period of deterioration probably coincided with transformation of the tumor into a gliosarcoma. The variable CT characteristics of gliosarcomas are reviewed.
\end{abstract}

Résumé: Évolution inhabituelle et aspect tomodensitométrique d'un gliosarcome. Un patient présentant un infarctus ancien, des convulsions, une légère hémiparésie et une dysphasie, a développé de la confusion quatre mois avant son décès. La tomodensitométrie sériée sur 5 ans a montré une zone étendue, de forme triangulaire, hypodense, sans calcification centrale, mais sans aspect de masse, à l'hémisphère gauche. Cette image a été interprétée comme étant un infarctus dans le territoire de l'artère cérébrale moyenne. L'autopsie du cerveau a révélé que toute la zone qui avait l'apparence d'un infarctus à la tomodensitométrie était en fait un gliosarcome. Nous soupçonnons que l'aspect inhabituel de la lésion à la tomodensitométrie était dû à des pathologies multiples: un gliome évoluant à bas bruit qui s'est transformé en gliosarcome et qui a envahi la zone d'encéphalomalacie post-infarctus, sans produire d'aspect de masse à la tomodensitométrie. La détérioration rapide du patient a probablement coïncidé avec la transformation de la tumeur en gliosarcome. Nous revoyons les caractéristiques tomodensitométriques variables des gliosarcomes.

Can. J. Neurol. Sci. 1994; 21: 141-145

Gliosarcomas account for $1.7 \%$ to $5 \%$ of astrocytic tumors..$^{1.4}$ Their clinical presentation and progression are similar to that of glioblastomas, although some reports suggest that gliosarcomas may be more aggressive. ${ }^{3,5}$ Survival is generally less than one year. ${ }^{3}$ The mechanism of induction of such tumors remains unknown, although it is generally believed that the sarcomatous component arises from neoplastic change in the vascular endothelium of a pre-existing glioblastoma multiforme. ${ }^{1,2,4,6,7}$

The radiological appearance of gliosarcomas is not well known. They may look similar to glioblastomas, or assume a more variable and heterogeneous appearance on computed tomography (CT). Both previous reports on the imaging of gliosarcomas by CT suggest that they are hyperdense on the non-contrast scan and markedly enhance with contrast infusion. ${ }^{8,9}$ We report an unusual case of a 59-year-old man with a history of seizures, mild hemiparesis and dysphasia for 5.5 years who became obtunded over 4 months and whose CT was interpreted as consistent with a large left hemisphere infarct, but at autopsy was found to have a large gliosarcoma in the area interpreted radiologically as infarct. We propose that in this patient multiple pathologies combined to form a lesion with an unusual CT appearance.

\section{CASE RePORT}

At age 49 , the patient was hospitalized because of the onset over 2 weeks of dysphasia and dysgraphia and then a right hemiparesis. This was considered to represent a CVA in the distribution of the left middle cerebral artery. He had a past history of smoking, hypertension, coronary artery disease with angina, and a left internal carotid bruit. On admission he was stuporous and had a right flaccid hemiplegia. Neither CT nor angiography were performed. After six weeks he had no cognitive deficits and was discharged with $3 / 5$ strength in the right lower limb.

At age 54, he had an episode of sudden loss of consciousness that was interpreted as a seizure resulting from a cortical scar. A CT scan (Figure I A and B) showed an old infarct in the left middle cerebral artery territory with a $2.5 \mathrm{~cm}$ focus of calcification within the area of infarct that was interpreted as

From the Divisions of Neurosurgery, Neuroradiology and Neuropathology, Montreal Neurological Institute and Hospital, McGill University, Montreal. RECEIVED FEBRUARY 16, 1993. ACCEPTED IN FINAL FORM NOVEMBER 12, 1993

Reprint requests to: Donatella Tampieri, MD, Division of Neuroradiology, Montreal Neurological Hospital, 3801 University Street, Montreal, PQ. Canada H3A 2B4 
resolution of a previous hematoma or granuloma. Review of his clinical state was consistent with an old CVA. There was no evidence of a space-occupying lesion. One year later, CT showed no change.

The patient did well and was largely able to care for himself at home with minimal assistance despite the events previously recorded.

Four years later, at age 59, he developed a Bell's palsy and complained of left frontal headaches which radiated to the left temporal and occipital areas. On CT (Figure $2 \mathrm{~A}$ and $\mathrm{B}$ ) no changes were evident in the left hemisphere hypodensity or the area of hyperdensity interpreted as calcification. EEG showed mild to moderate slowing activity without any epileptiform features over the left hemisphere reaching a maximum in the left anterior quadrant. There remained no shift of the midline stuctures. During this hospital admission he had a fall near his bed with a few episodes of nausea and vomiting following for 3 days that were attributed to cholelithiasis and a partially obstructed common bile duct.

During the ensuing 4 months, for unknown reasons, his clinical status gradually deteriorated and he died. A CT scan of the brain 2 months before his death (Figure $3 \mathrm{~A}$ and $\mathrm{B}$ ) was unchanged compared to previous studies. He never had MR imaging.

A.

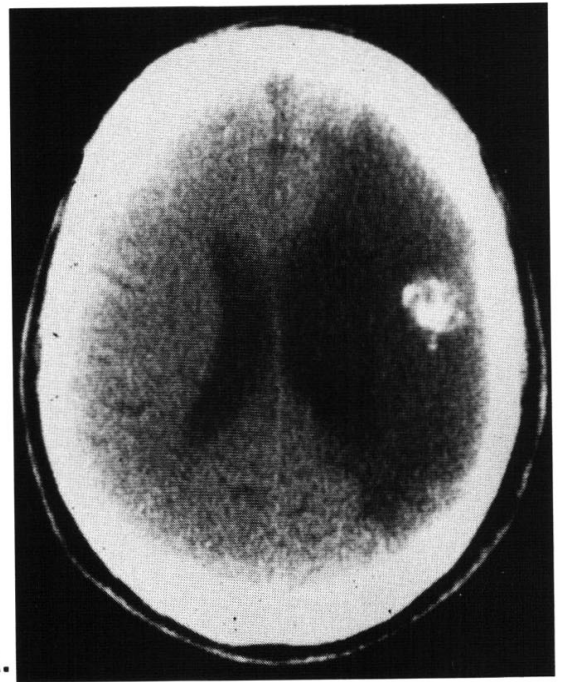

B.

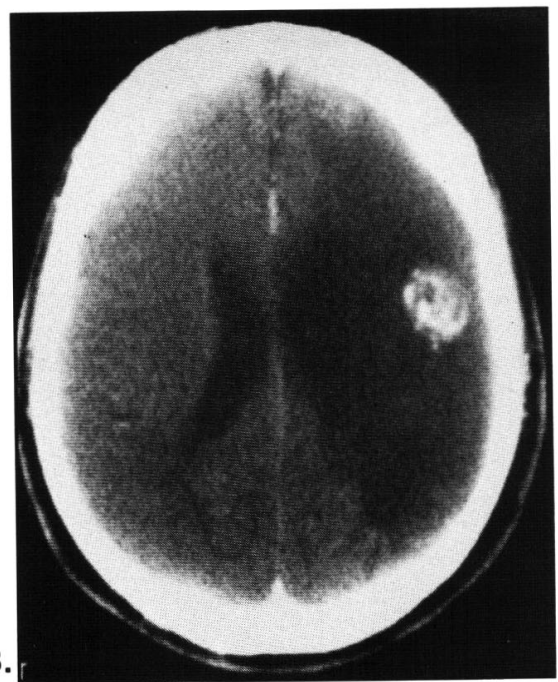

Figure 1: $C T$ without $(\boldsymbol{A})$ and with $(B)$ contrast infusion at the time of seizure onset, 5 years after a radiologically undocumented infarct. A large, wedge-shaped hypodensity extends from the left frontal area to the left posterior parietal area. The left lateral ventricle shows ex vacuo enlargement and the $2.7 \mathrm{~cm}$ hyperdense area is consistent with calcification in the left posterior frontal area.

\section{Post-mortem Brain Examination}

A post-mortem examination revealed a brain weight of 1600 grams, due to the large and extremely dense, grossly fibrotic mass in the left fronto-centro-parietal convexity (Figure 4). At the base there was moderately severe arteriosclerosis with $50 \%$ stenoses in the vertebral and distal basilar arteries associated with $20 \%$ stenoses in other areas of the circle of Willis. Transtentorial herniation was not present and the cerebellar tonsils were not herniated. Brain tissue surrounding the mass was soft and boggy.

The tumor measured $7.5 \times 5 \times 6.5 \mathrm{~cm}$, and was partly soft yellow with pink to red streaks and in other parts pale gray, firm, glistening and homogenous. It involved the left frontal and temporal lobes and posterior parietal area (protruding into the subarachnoid space), the deep white matter, cingulum, caudate,
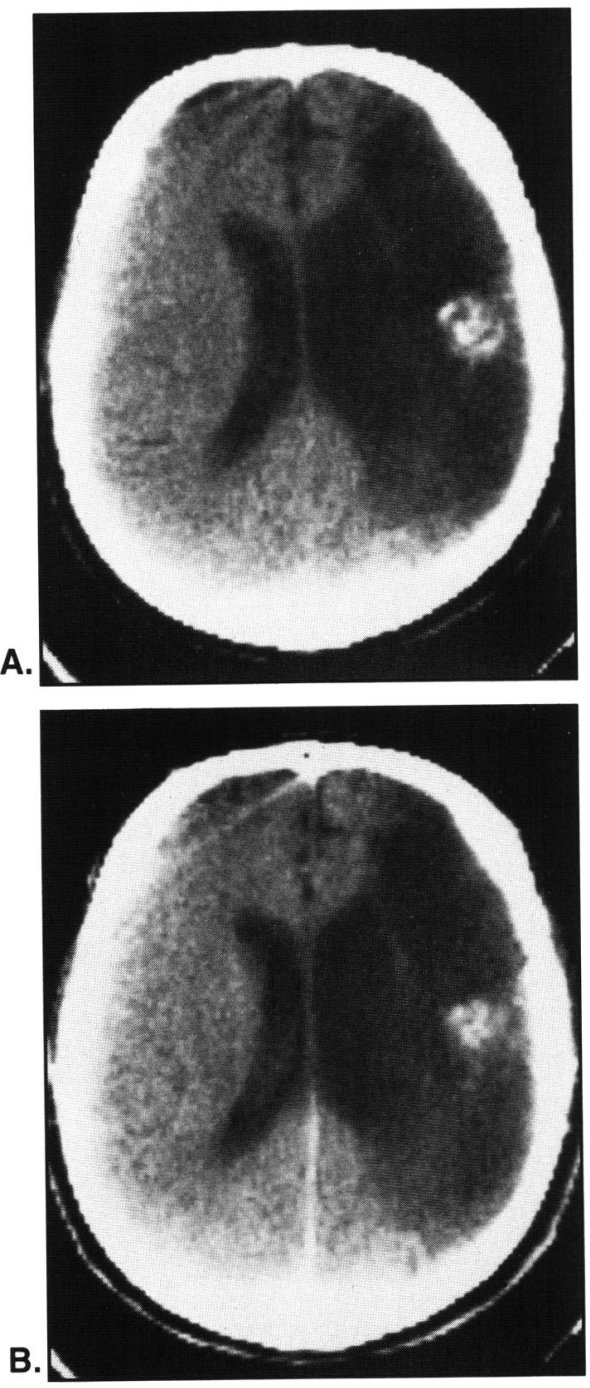

Figure 2: $C T$ scan without $(A)$ and with $(B)$ contrast infusion at the time of onset of Bell's palsy and headaches, 4 years after the CT scan depicted in Figure 1 , interpreted as showing natural interval changes consistent with an old large ischemic area. Although slightly lower cuts, the area of calcification and the large, wedge-shaped hypodensity are similar in appearance to Figure I. There are no other hyperdense or enhancing features and there is no tumoral edema or mass effect. 
lenticular nucleus, thalamus, and meninges. The left ventricle was moderately enlarged, and the ependymal surface grossly invaded by tumor. In the posterior frontal area there was a 2.7 $\mathrm{cm}$ diameter, spherical, whitish, calcified area that corresponded to the hyperdense area on CT. The tumor was not dural-based, although the meninges overlying the area of the tumor throughout the hemisphere were thickened and difficult to separate from the brain. The normal gyral-sulcal pattern of the left convexity was completely obliterated by the tumor so that the periphery of the brain appeared nearly smooth. Tumor surrounded the left third cranial nerve. The stem of the left middle cerebral artery was markedly compressed by the tumor, and the basilar artery showed $50 \%$ narrowing of its lumen by atherosclerosis.

Cross-sections of the brain stem showed an atrophic basis pontis. Sections through the cerebellum showed pale white matter with yellowish discoloration around the dentate nucleus. An old

A.

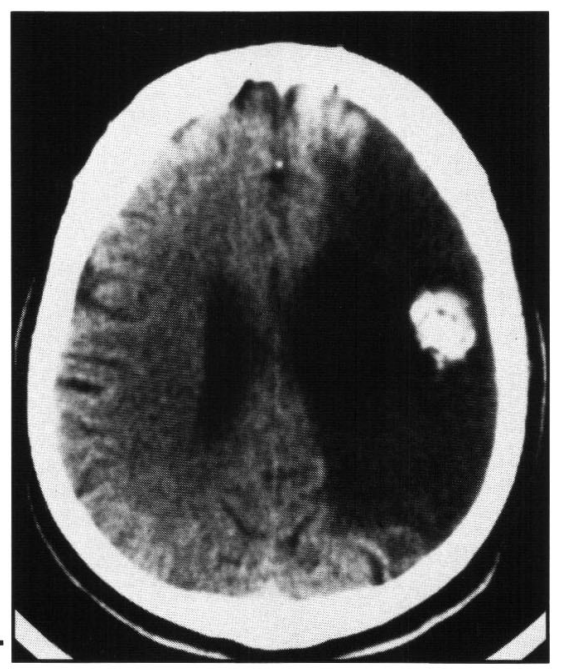

B.

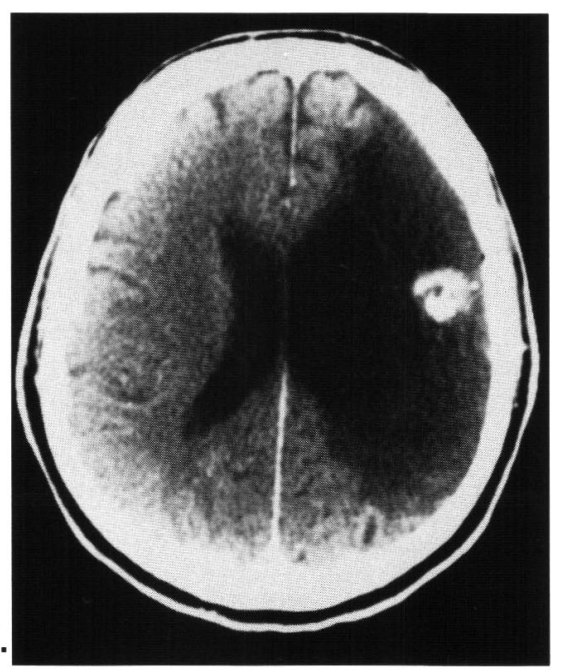

Figure 3: $C T$ scan without $(A)$ and with $(B)$ contrast infusion at the time of neurologic deterioration, 2 months before death (nearly 5 years after the CT scan in Figure 1). The hypodense area interpreted as infarct and the area of calcification are unchanged compared to Figures $I$ and 2. There are no enhancing structures, tumoral edema, or mass effect. The gliosarcoma occupied the entire territory identified as an infarct.

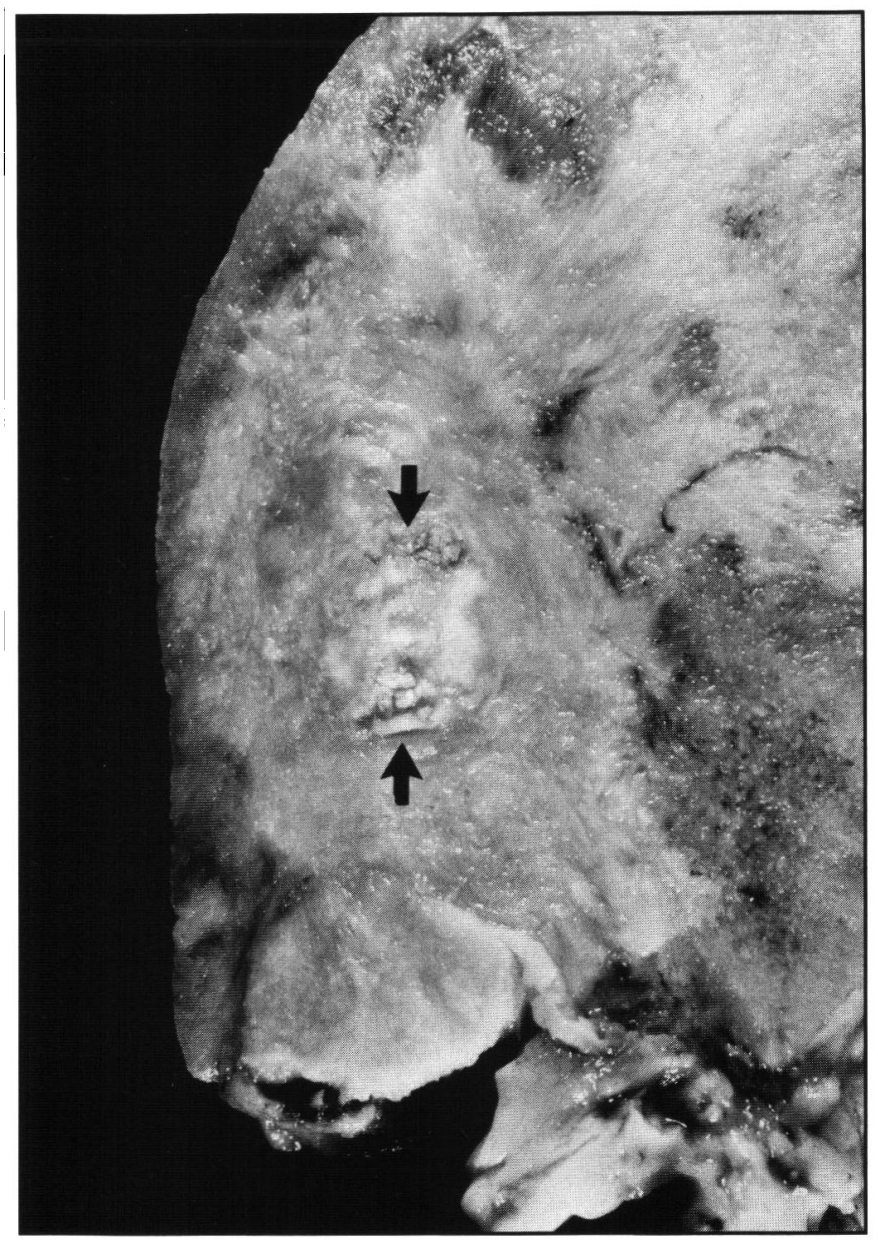

Figure 4: Photograph of the left posterior frontal and temporal lobes in coronal section at gross brain examination. Note the smooth contour of the brain mantle; there is complete disruption of the normal gyral-sulcal pattern. There is no normal brain: it is extremely dense, grossly fibrotic, and completely comprised of gliosarcoma. An area, $2.7 \mathrm{~cm}$ in diameter, of calcification (admixed with gliosarcoma), noted on CT, is within the arrows. The left lateral ventricle (not shown) revealed evidence of ex vacuo enlargement. Although tumor filled nearly the entire left hemisphere, there was no mass effect, presumably because the tumor had expanded in a previous area of encephalomalacia due 10 an infarct.

infarct $(4 \times 2 \mathrm{~cm}$ diameter) involved the superior and inferior parietal cerebellar lobule.

Microscopic sections (hematoxylin and eosin, reticulin, glial fibrillary acidic protein) revealed the appearance of anaplastic astrocytes intermingled among nodules and thick strands of perivascular, pleomorphic, spindle-shaped fibrosarcomatous cells with large areas of collagen (Figure $5 \mathrm{~A}$ and B). Glial fibrillary acidic protein showed strong positivity within relatively limited areas of the tumor. In most areas the strands of malignant glial tissue were encompassed by the sarcomatous tissue. Sarcomatous tumor had completely filled the lumen of a moderately-sized artery within the tumor (Figure 6). The tumor was sparsely vascularized.

The area of calcification (Figure 7) was located within an area of necrotic debris with numerous cholesterol clefts and 
surrounded by a fibrous capsule and sarcoma. It appeared to represent an old area of brain necrosis which had escaped phagocytosis.

The cortex adjacent to the tumor revealed a segmental infarction with cavitation involving all cortical layers except the molecular layer which displayed a coarse gliosis. Residual macrophages were found within the cavity which was infiltrated by tumor.

\section{Discussion}

Gliosarcomas comprise $1.8 \%$ to $8 \%$ of glioblastomas. ${ }^{10,11}$ They are composed of malignant glial cells in association with fibrosarcoma. The term "gliosarcoma" came into use by Stroebe in 1895 to describe such a tumor, however, during the next three decades "gliosarcoma" was used to describe tumors that are now called anaplastic astrocytomas, or those tumors with a high degree of endothelial hyperplasia. ${ }^{12}$ The report by Morantz, et al., in 1976 re-introduced "gliosarcoma" to describe the presently recognized mixed glioblastoma multiforme and sarcoma. $^{5}$

In most instances, the initiation of gliosarcomas is due to extreme endothelial hyperplasia in high grade gliomas.
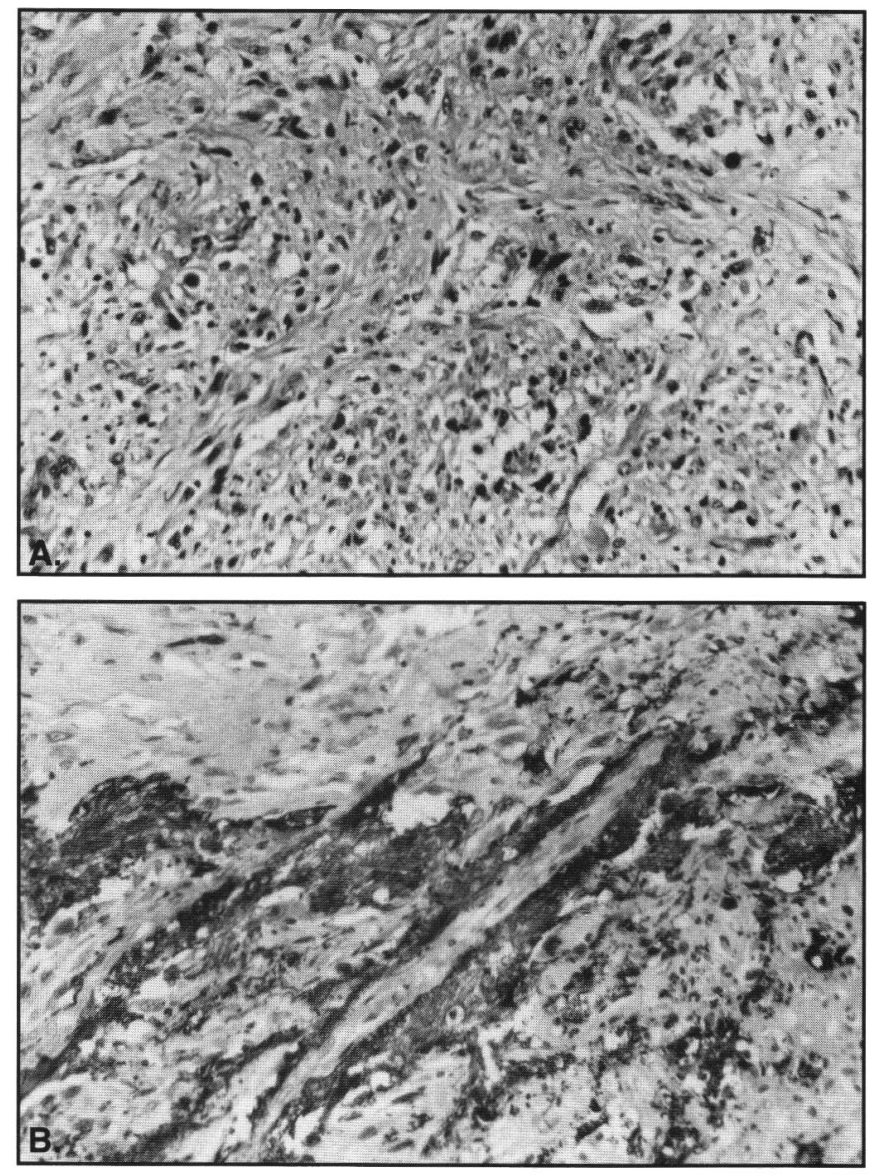

Figure 5: A. Photomicrograph showing an area of tumor with interlacing bundles of pleomorphic cells, some of which are sarcomatous and others astrocytic (hematoxylin-eosin, 180x). B. This field shows GFAPpositive areas (dark areas) intermingling with GFAP-negative sarcomatous areas (immunocytochemistry, avidin-biotin-peroxidase, 180x).

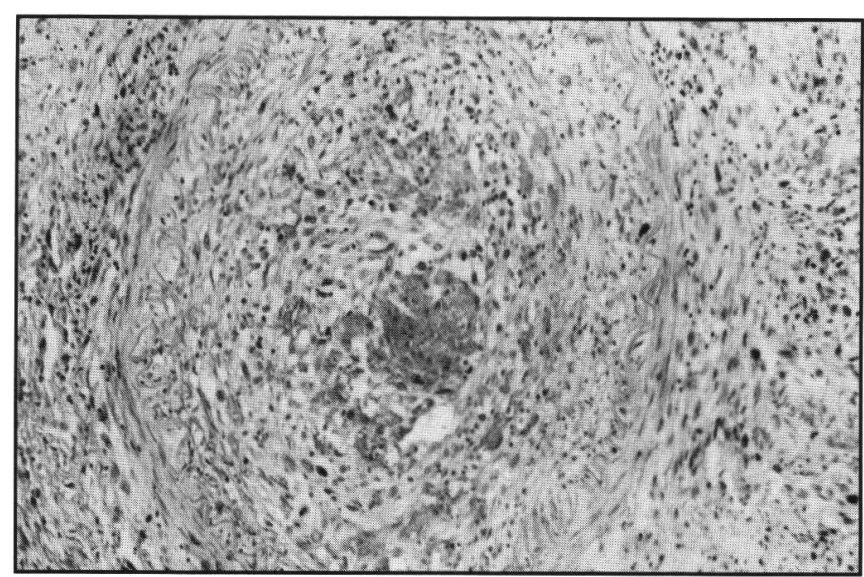

Figure 6: Sarcoma fills the lumen of a moderately-sized artery in the parietal lobe $(100 x)$.

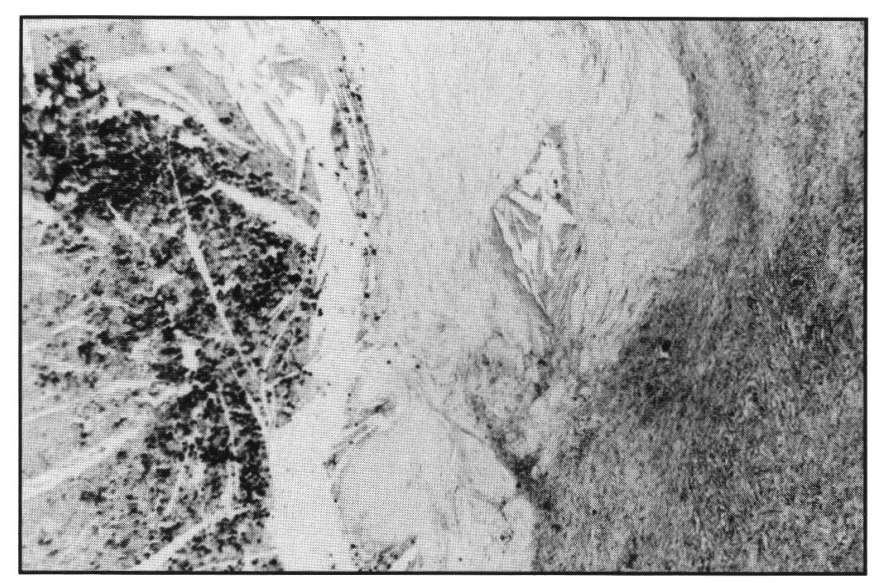

Figure 7: Photomicrograph of a section through the area of calcification. On the left, calcified debris stains strongly and cholesterol clefts are present. On the right is a fibrous capsule surrounding the area of calcification. Tumor outside the fibrous capsule is seen in the lower $\operatorname{right}(50 x)$.

However, this process goes beyond mere hyperplasia and assumes neoplastic change with subsequent invasion of the surrounding tissues. The malignant endothelial cells assume the appearance of a spindle-cell fibrosarcoma and arrange themselves in thick, parallel rows, or form rounded nodules that may encircle the glial component. Factor VIII-related antigen staining indicates that the mesenchymal component of gliosarcomas may be of endothelial origin. ${ }^{13.14}$ At times the sarcomatous tissues may outgrow the glial component and form the bulk of the tumor.

There have, however, been cases described where the sarcoma and glioma arise de novo at the same time and become mixed as the tumor expands. ${ }^{11}$ Further confusing the origin of these tumors are those cases in which a sarcoma is the initial tumor and induces a surrounding glioma ("sarcoglioma"). ${ }^{15}$

Gliosarcomas have a clinical progression and prognosis similar to glioblastomas. ${ }^{5.12}$ Survival time ranges from 24 to 37 weeks from onset of symptoms in patients surviving operation at least 5 weeks. ${ }^{5,16}$ 


\section{CT Appearance of Gliosarcomas}

Computed tomography of gliosarcomas has only recently been described in three reports that address their characteristics in 24 patients, although in only 10 is the CT appearance documented. ${ }^{8,9,17}$ In most cases, non-contrast CT showed hyperdense lesions due to the high cellularity and vascularity. Contrastinfused CT has demonstrated an appearance similar to glioblastomas, with central areas of hypodensity corresponding to necrosis, and heterogeneous peripheral enhancement. Gliosarcomas may appear similar on CT to meningiomas when in contact with the skull or falx. They usually also have a more sharply demarcated appearance from surrounding brain than glioblastomas. Peritumoral edema is suggested to be ubiquitous. The lesions may be more peripherally located and have a tendency to invade the dura more than other gliomatous tumors. This supports data indicating that gliosarcomas are more aggressive than glioblastomas, as gliosarcomas have a much greater tendency to metastasize than other brain tumors. ${ }^{3.5}$

Although we believe the pathology progressed from ischemic event to low grade glioma to gliosarcoma, the original CT appearance of the brain of our patient remained consistent. The vast majority of gliosarcomas will enhance with contrast, and a minority may show calcification. Also, the vascularization of gliosarcomas may vary with the content of the fibrosarcomatous component, causing the tumor to be a non-enhancing entity with contrast infusion. Thus, they may demonstrate a variable CT appearance, similar to an infarct.

\section{Unusual CT Features Caused by Multiple Pathologies}

More than one pathology is responsible for the unusual imaging appearance of this lesion. In our patient, we conjecture that the previous left middle cerebral artery territory infarction and the presence of a second pathological process of a longstanding low grade glioma produced complicated imaging features of this unusual gliosarcoma. We believe that a low grade glioma developed in the area of previous infarct and then underwent malignant sarcomatous transformation a few months prior to the patient's death. The calcified area suggests the presence of a long-standing, low grade glioma. We suspect that the tumor was able to enlarge and spread throughout the area of encephalomalacia, as a result of the infarct, without causing the usual clinical sequelae of mass effect. The relatively brief, but rapid, period of neurological deterioration presumably coincided with the transformation into a gliosarcoma. However, the consistent appearance of the brain on CT over the years did not give an impression of an expanding intracranial mass. Although the brainstem did not show signs of herniation at post-mortem examination, neurologic deterioriation may have been hastened by the compression of the left middle cerebral artery by the tumor. The exact cause of death, however, cannot be determined because a complete autopsy was not performed.
Diagnosis of this case was difficult on a clinical basis as CT did not provide evidence of the gliosarcoma, although the natural evolution of a large ischemic area is typically not one of deterioration with new neurological signs. The unusual appearance of this patient's gliosarcoma is predominantly related to the previous underlying middle cerebral artery infarction. Inconsistent clinical presentation with an entity such as an atypical infarct with calcification necessitates further evaluation. An MRI would likely have defined the tumor and disruption of the gyral pattern noted at the post-mortem brain examination.

\section{REFERENCES}

1. Feigin IH, Allen LB, Lipkin L. The endothelial hyperplasia of the cerebral blood vessels with brain tumors and its sarcomatous transformation. Cancer 1958; 11: 264-277.

2. Feigin IH, Gross SW. Sarcoma arising in glioblastoma of the brain. Am J Pathol 1955; 31: 633-653.

3. Morantz RA, Feigin I, Ransohoff J. Clinical and pathological study of 24 cases of gliosarcoma. J Neurosurg 1976; 45: 398-408.

4. Wislowski J. Gliosarcoma tumors with a sarcomatous neurogliosarcoma and glioma multiforme in a neoplastic area. Neuropathol Pol 1970; 8: 23-28.

5. Smith DR, Hardman JM, Earle KM. Contiguous glioblastoma multiforme and fibrosarcoma with extracranial metastasis. Cancer 1969; 24: 270-276.

6. Goldman RL. Gliomyosarcoma of the cerebrum. Am J Clin Pathol 1969; 52: 741-744.

7. Greene HSN, Harvey EK. The development of sarcomas from transplants of the hyperplastic stromal endothelium of glioblastoma multiforme. Am J Pathol 1968; 53: 483-499.

8. Lee Y-Y, Castillo M, Nauert C, Moser RP. Computed tomography of gliosarcoma. Am J Neuroradiol 1985; 6: 527-531.

9. Maiuri F, Stella L, Benvenuti D, Giamundo A, Pettinato G. Cerebral gliosarcomas: correlation of computed tomographic findings, surgical aspect, pathological features, and prognosis. Neurosurgery 1990; 26: 261-267.

10. Meis JM, Martz KL, Nelson JS. Mixed glioblastoma multiforme and sarcoma. A clinicopathologic study of 26 radiation therapy oncology group cases. Cancer 1991; 67: 2342-2349.

11. French JD. Astroblastoma and perithelial sarcoma in a case of neoplastic disease of the brain. J Neuropathol Exp Neurol 1949;8: 232-239.

12. Stroebe H. Uber Entstehung und Bau der Gehirngliome. Beitr Pathol 1895; 18: 405-486.

13. Schiffer D, Giordana MT, Mauro A, Migheli A. GFAP, F VIIl/RAg, laminin, fibronectin in glioscarcomas: an immunohistochemical study. Acta Neuropathol 1984; 63: 108-116.

14. Slowik F, Jellinger K, Gas zo L, Fischer J. Gliosarcomas: histological, immunohistochemical, ultrastructural, and tissue culture studies. Acta Neuropathol 1985; 67: 201-210.

15. Lalitha VS, Rubinstein LJ. Reactive glioma in intracranial sarcoma: a form of mixed sarcoma and glioma ("sarcoglioma"). Cancer 1979; 43: 246-257.

16. Goldstein SJ, Young B, Markesberry WR. Congenital malignant gliosarcoma. Am J Neuroradiol 1981; 2: 475-476.

17. Jack CR, Bhansali DT, Chason JL, et al. Angiographic features of gliosarcoma. Am J Neuroradiol 1987; 8: 117-122. 